\title{
Factores biológicos y psicosociales predictores del estilo de vida promotor de salud
}

\author{
Patricia Cid $\mathrm{H}^{\mathrm{1a}}$, José Manuel Merino $\mathrm{E}^{2 \mathrm{~b}}$, \\ Jasna Stiepovich Bc. \\ Biological and psychosocial factors \\ that predict health promoting lifestyles
}

Background: One of the goals of the national health reform is to promote a healthy lifestyle in all the population. The determinants of healthy lifestyles need to be identified to guide people towards this purpose. Aim: To analyze the health promoting lifestyle (HLP) in a community of Concepción and to identify the personal factors that might be predictors of such lifestyle. Material and methods: Pender's health promotion model was used as the conceptual framework for exploring the HLP among 360 persons aged from 15 to 64 years, free from chronic diseases. Data were obtained by a semi structured questionnaire and the following scales were used to measure HLP: perceived health status, perceived self esteem, and perceived self efficacy. Relationships between personal factors and HLP were studied. Data were processed with SAS software and analyzed with descriptive and inferential statistics. Results: Only $52.2 \%$ of the sample had a healthy lifestyle. The variables sex, age, occupation, access to health care, self esteem, perceived health status and perceived self efficacy had a significant effect on HLP. A regression model showed that self esteem, self efficacy, sex, age, occupation and access to health care explained $30 \%$ of the variation in health lifestyle. Conclusions: Only half of the studied sample had a healthy lifestyle. The variables obtained with the regression model, should be considered to create effective interventions encouraging persons to integrate healthy behaviors to their life (Rev Méd Chile 2006; 134: 1491-99).

(Key w ords: Life style; Psychosocial factors; Regression analysis)

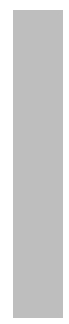

Recibido el 26 enero, 2006. Aceptado el 31 de mayo, 2006.

Proyecto de la Dirección de investigación. Universidad de Concepción Concurso 2002 №202.082.029-1. Sin influencia en el desarrollo de la investigación y preparación del manuscrito.

${ }_{1}^{1}$ Departamento de Enfermería, Facultad de Medicina, Universidad de Concepción, Chile.

${ }^{2}$ Facultad de Ciencias Sociales, Universidad de Concepción, Chile.

aEnfermera, Magíster

bSociólogo, Ph D.

'Enfermera, Doctor en Enfermería

Correspondencia a: Patricia Cid H. Departamento de Enfermería, Facultad de Medicina, Universidad de Concepción. Dirección: Casilla 160-C. Fax: 228353 Teléfonos: 2207064 - 2207065.

E mail: patcid@udec.cl 
$E^{\mathrm{n}}$ la VI Conferencia Internacional sobre la Promoción de la Salud realizada el año 2005, en Islandia, se reafirmó el aspecto positivo de la promoción de la salud, estableciendo que "consiste en capacitar a la gente para mejorar su salud ejerciendo un mayor control sobre sus factores influyentes", concepto establecido en la Carta de Ottawa en 1986. Desde la adopción de la Carta de Ottawa, se ha suscrito varias resoluciones en el ámbito nacional y mundial en apoyo de la promoción de la salud, pero esas resoluciones no siempre han tenido los efectos correspondientes ${ }^{1}$.

Nuestro país adoptó las metas y directrices internacionales de la Organización Mundial de la Salud (OMS) acerca de la promoción de salud. En esa perspectiva, el Ministerio de Salud formuló los objetivos sanitarios para la década 2000-2010, con la finalidad de detener la explosión de factores de riesgo y desarrollar factores protectores de la salud de carácter psicosocial y ambiental. En ese contexto, en 2003 se inició una reorientación de los servicios sanitarios hacia la promoción de salud y prevención de enfermedades, cambio cultural orientado a crear conciencia en la población nacional de la autorresponsabilidad que cada persona tiene respecto de su salud, "que motive y oriente hacia conductas saludables por la vía de modificación de estilos de vida no saludables"2,3.

La aplicación de este enfoque es una tarea compleja, porque incrementar las actividades saludables o disminuir las que determinan riesgo no es algo fácil, puesto que significa cambios de conductas, que, a su vez, implican gatillar estados de insatisfacción como resultado de reconocer inconsistencias entre las propias creencias, valores, y conductas 4 .

Esta investigación tuvo como propósito principal, analizar el estilo de vida promotor de salud de las personas que residen en el sector Barrio Norte de Concepción e identificar algunas orientaciones culturales que resultan predictoras de este estilo de vida, para desarrollar estrategias dirigidas a provocar en los sujetos las motivaciones necesarias para integrar conductas positivas a sus estilos de vida.

Conceptualmente, este estudio se sustenta en el Modelo Promotor de Salud de Pender. Este modelo (Figura 1) es un esquema que integra constructos de la teoría de expectativas-valores y de la teoría cognitivo-social, para detectar la estructura multidimensional de los rasgos individuales que, en interacción con su ambiente, mejoran los resultados asociados a salud. La conducta promotora de salud es un resultado determinado por características y experiencias individuales previas, y por cogniciones y afectos relativos a comportamientos específicos ${ }^{5}$.

\section{MATERIAL Y MÉTODOS}

Esta investigación es un estudio correlacionaltransversal efectuado en residentes del sector Barrio Norte de Concepción, en el segundo semestre de $2002^{6}$. El universo lo constituyeron 18.353 personas inscritas en el registro del Servicio de Salud del sector, conformándose una muestra de 360 sujetos de ambos sexos, de 15 a 64 años. Se utilizó muestreo por conglomerado y estratificado al azar, a porcentaje proporcional, siendo la edad el criterio de estratificación.

La variable dependiente estudiada fue el estilo de vida promotor de salud (EVPS) que se define como las actitudes o conductas que desarrolla una persona con el objeto de incrementar su nivel de bienestar y que son parte regular de sus patrones de vida. Operacionalmente, fue medida con la escala EVPS, instrumento que permite analizar los puntajes obtenidos en cada dimensión (autorrealización, responsabilidad en salud, ejercicio, nutrición, apoyo interpersonal y manejo del estrés), de este modo identifica específicamente tanto los aspectos positivos como deficitarios del EVPS y también permite obtener un puntaje total para EVPS. La puntuación total va de 48 a 192: mientras más alto el puntaje total, mejor es el EVPS de la persona. La puntuación empírica para la escala total se calcula aditivamente con las respuestas dadas por las personas entrevistadas a los 48 ítems.

La recolección de los datos se realizó a través de un instrumento de medición conformado por un cuestionario semiestructurado, y por las escalas: Estilo de Vida Promotor de Salud7; Autoestima ${ }^{8}$; Estado de Salud Percibido y Percepción de Autoeficacia $^{9}$. El instrumento se aplicó a través de una entrevista personalizada en la residencia de las personas (quienes dieron su consentimiento informado para participar). 


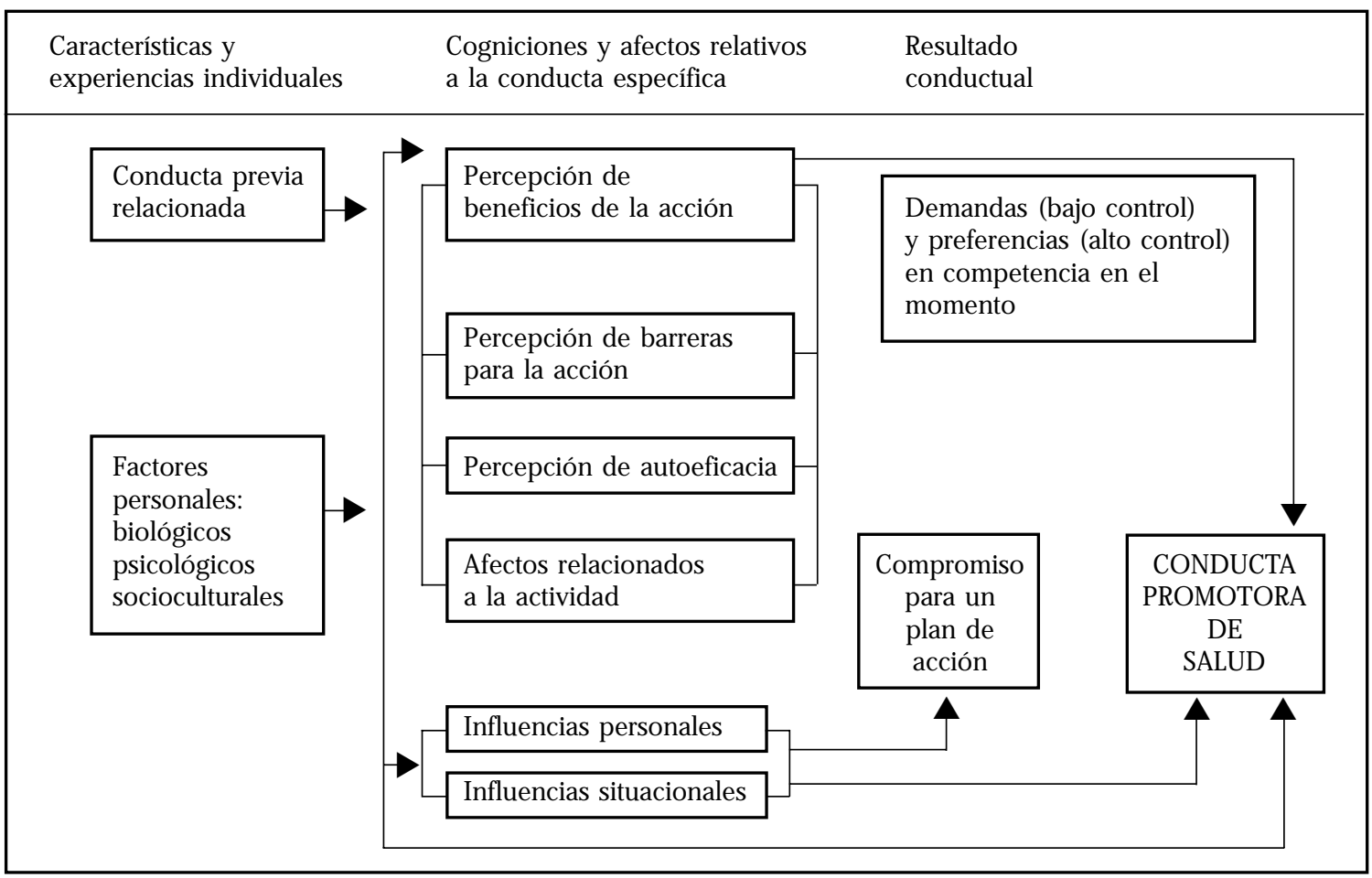

Figura 1. Modelo de Promoción de Salud de Nola Pender, 1996.

La confiabilidad de las escalas se midió con el coeficiente Alpha de Cronbach y el método de división en mitades. El resultado de ambas pruebas indica alta confiabilidad $(0,73$ a 0,87$)$.

En el procesamiento y análisis estadístico se usó el programa Statistical Analysis System (SAS). Se aplicó estadística descriptiva e inferencial, usando $\mathrm{p}<0,05$ como el valor de la probabilidad del error en las pruebas de hipótesis estadísticas.

\section{RESULTADOs}

La distribución empírica de los puntajes obtenidos de la variable dependiente EVPS fue la siguiente (Tabla 1): el mínimo fue de 78 puntos y el máximo de 183 puntos, siendo la media de 131,5 puntos $(52,2 \%$ de las personas obtuvieron puntajes iguales o mayores a la media) y la mediana de 132 puntos. Las medidas de tendencia central son muy cercanas, por lo que puede decirse que la distribución tiene una asimetría mínima. El 25\% de las personas obtuvo puntajes inferiores a 119 puntos y otro $25 \%$ alcanzó puntajes superiores a 146 puntos en la escala EVPS.

\section{Tabla 1. Estadísticas descriptivas de la variable Estilo de Vida Promotor de Salud}

\begin{tabular}{lr} 
Escala & \\
Estilo de Vida Promotor de Salud & \\
\hline Mínimo & 78,0 \\
Máximo & 183,0 \\
Media (x) & 131,5 \\
Error típico de la media & 0,9 \\
Mediana & 132,0 \\
Moda & 125,0 \\
Desviación típica & 18,8 \\
Asimetría & $-0,2$ \\
Curtosis & $-0,1$ \\
Rango & 105,0 \\
Cuartil 1 & 119,0 \\
& 132,0 \\
\multicolumn{1}{c}{2} & 146,0
\end{tabular}


En síntesis, esta variable tuvo una distribución relativamente simétrica.

Atributos de la muestra (Tabla 2): Predominó el sexo femenino, la edad mínima fue de $15 \mathrm{y}$ la máxima de 64 años. En promedio, las personas tenían 11 años de estudios. Sus ingresos familiares oscilaron desde $\$ 30.000$ a $\$ 999.999$. Un poco más de la mitad declaró profesar la religión católica, y una de cada tres personas adhería a la religión evangélica. El 20\% realizaba actividades de comerciantes, empleados o trabajadores independientes y un tercio eran dueñas de casa o estudiantes. Un poco más de la mitad se calificó con buena autoestima, buen estado de salud y autoeficaces.

Al relacionar cada uno de los factores personales con la variable EVPS (Tabla 3), se obtuvieron relaciones significativas de la variable dependiente con las variables sexo, edad, acceso a salud, ocupación, estado de salud percibido, autoeficacia y autoestima.

La Tabla 3 indica que las variables escolaridad, religión, estado marital, e ingresos, en este estudio, no tienen efectos independientes sobre el EVPS por lo que deben ser excluidos de análisis posteriores.

Cuando se compara el sexo y EVPS se encuentra que: las mujeres tenían un estilo de vida más promotor de salud que los hombres. El promedio de EVPS en mujeres fue de 134,5 puntos y los hombres tuvieron en promedio siete puntos menos, diferencia estadísticamente significativa al seis por diez mil.

La edad de las personas también resultó un predictor significativo de su EVPS: existió una relación positiva entre la edad y el puntaje EVPS total, esto es, a mayor edad puntajes más altos en la

Tabla 2. D escripción de los factores personales de los residentes de Barrio N orte

\begin{tabular}{|c|c|c|c|c|c|}
\hline \multicolumn{2}{|c|}{ Factores personales } & \multirow{3}{*}{$\begin{array}{c}\text { Media } \\
0,6 \\
0,4\end{array}$} & \multirow{2}{*}{$\frac{\text { ds }}{0,5}$} & \multirow[t]{2}{*}{ Mín } & \multirow[t]{2}{*}{ Máx } \\
\hline Sexo & Femenino (0=no; 1=sí) & & & & \\
\hline & Masculino (0=no; 1=sí) & & & & \\
\hline \multirow[t]{3}{*}{ Edad } & $<35$ años $(0=$ no; $1=$ sí) & 129,2 & & 15 & 65 \\
\hline & 35-55 años (0=no; 1=sí) & 133,0 & & & \\
\hline & $>55$ años $(0=$ no; $1=$ sí) & 139,2 & & & \\
\hline Escolaridad & Años de estudios & 11,0 & 3,8 & 0 & 20 \\
\hline Ingreso familiar & $\begin{array}{l}\text { Suma de los ingresos en pesos } \\
\text { de los integrantes de la familia }\end{array}$ & 217,2 & 166,8 & 30000 & 999999 \\
\hline \multirow[t]{2}{*}{ Estado marital } & Con pareja $(0=n 0 ; 1=$ sí) & 0,6 & 0,5 & & \\
\hline & Sin pareja $(0=n o ; 1=s i ́)$ & 0,4 & & & \\
\hline \multirow[t]{3}{*}{ Religión } & Católica $(0=\mathrm{no} ; 1=\mathrm{sí})$ & 0,5 & 0,5 & & \\
\hline & Evangélica (0=no; 1=sí) & 0,4 & 0,5 & & \\
\hline & Sin religión & 0,1 & 0,3 & & \\
\hline \multirow[t]{6}{*}{ Actividad laboral } & Profesionales $(0=$ no; $1=$ sí) & $<0,1$ & 0,2 & & \\
\hline & Independientes ( $0=$ no; $1=$ sí) & 0,2 & 0,4 & & \\
\hline & Obreros $(0=$ no; $1=s i ́)$ & 0,1 & 0,2 & & \\
\hline & Inactivos $(0=$ no; $1=$ sí) & 0,2 & 0,4 & & \\
\hline & Dueñas de casa $(0=$ no; $1=$ sí) & 0,3 & 0,5 & & \\
\hline & Cesantes $(0=\mathrm{no} ; 1=\mathrm{sí})$ & 0,1 & 0,3 & & \\
\hline \multirow[t]{2}{*}{ Acceso a salud } & Sí $(0=$ no; $1=$ sí) & 0,9 & 0,3 & & \\
\hline & No $(0=$ no; $1=$ sí) & 0,1 & & & \\
\hline Autoestima & & 21,7 & 4,2 & 15 & 40 \\
\hline Autoeficacia & & 34,2 & 4,9 & 16 & 40 \\
\hline \multirow{2}{*}{\multicolumn{2}{|c|}{$\begin{array}{l}\text { Estado de salud } \\
\text { percibida }\end{array}$}} & & & & \\
\hline & & 82,8 & 13,4 & 45 & 112 \\
\hline
\end{tabular}


Tabla 3. Regresión bivariada de cada factor del estudio con Estilo de Vida Promotor de Salud

\begin{tabular}{|lccc|}
\hline Variables & gl & $\mathrm{R}^{2}$ & $\mathrm{p}$ \\
\hline Sexo: hombres & 1 & 3,3 & $<0,001$ \\
Edad: (35-55) + (> 55) & 2 & 1,8 & $<0,05$ \\
Escolaridad: baja + media & 2 & 1,2 & $\mathrm{NS}$ \\
Religión: católica + evangélica & 2 & 0,0 & $\mathrm{NS}$ \\
Estado marital: con pareja & 1 & 0,0 & $\mathrm{NS}$ \\
Ingreso & 1 & 0,0 & $\mathrm{NS}$ \\
Salud: sí acceso & 1 & 5,7 & $<0,0001$ \\
Ocupación: & & & \\
Profesionales + independientes + obreros + & 5 & 5,9 & $<0,001$ \\
inactivos + dueñas de casa & 1 & 3,4 & $<0,001$ \\
Estado de salud percibida & 1 & 9,6 & $<0,0001$ \\
Autoeficacia & 1 & 13,2 & $<0,0001$ \\
Autoestima & & & \\
\hline
\end{tabular}

escala EVPS. Las personas menores de 35 años obtuvieron 129,2 puntos, las de 35 a 55 tuvieron en cambio casi cuatro puntos más, mientras que las mayores de 55 alcanzaron el puntaje más alto con una diferencia de más de seis puntos. La diferencia de puntaje de EVPS fue significativa al tres por cien.

Al relacionar acceso a salud con EVPS, resultaron diferencias estadísticamente significativas en los promedios obtenidos, observándose que las personas con acceso a salud obtuvieron una media de 133,2 puntos, en tanto las personas sin acceso obtuvieron un promedio de 119,4 en la escala, esto es, catorce puntos menos.

De acuerdo a los valores estimados para los diferentes grupos de ocupación se puede apreciar que los profesionales tenían un promedio de 136,9, similar puntaje obtuvo el grupo de las dueñas de casa $(136,1)$, le siguen el grupo de los inactivos $(133,3)$ y el grupo de las personas que realizan trabajos de nivel medio $(132,7)$, lo que revela un EVPS semejante a los anteriores pero bastante diferente al del grupo de cesantes $(124,4)$ y del grupo de obreros y otros $(123,0)$ que manifestaban un EVPS más bajo, con diferencias estadísticamente significativas al seis por diez mil.

La autoestima resultó ser un importante predictor del EVPS. Su efecto fue, positivo: a medida que aumenta la autoestima, más alto es el nivel de EVPS. El efecto detectado indica que la autoestima explica el $13,2 \%$ de la variación total de la variable depen- diente EVPS. En términos de la ecuación de regresión, la escala EVPS expresa un aumento de 1,6 puntos por cada unidad de aumento en autoestima.

La relación entre autoeficacia y EVPS indica que a medida que las personas aumentan su percepción de autoeficacia aumentan también su EVPS. El 9,6\% de la variación del EVPS se debe a la percepción de autoeficacia. Otro predictor que tiene efectos positivos sobre el EVPS es el estado de salud percibido, indica que en tanto las personas perciben mejor su estado de salud, también mejora su EVPS, relación estadísticamente significativa al cinco por diez mil. Al examinar el coeficiente de determinación, se puede indicar que esta variable explica $3,4 \%$ la variación del EVPS. Las variables de la Tabla 3, con efectos independientes significativos, fueron posteriormente clasificadas para efectos analíticos en tres bloques conceptuales: variables sociodemográficas (sexo y edad), variables socioeconómicas (ocupación, acceso a salud) y orientaciones subjetivas (estado de salud percibido, percepción de autoeficacia y autoestima). En el bloque I (Tabla 4) se destaca que el modelo combinado de las variables sexo y edad con EVPS fue altamente significativo explicando $5,1 \%$ de la variación del EVPS. Una variación de $10,6 \%$ del EVPS es explicado por el bloque de las variables socioeconómicas, con una significancia estadística inferior al uno por diez mil. También resulta significativa 
Tabla 4. Predictores personales significativos del Estilo de Vida Promotor de Salud

\begin{tabular}{|lccc|}
\hline Bloques & gl & $\mathrm{R}^{2}$ & $\mathrm{p}$ \\
\hline Bloque de variables sociodemográficas & & & \\
Hombres & 1 & & $\mathrm{~N}$ \\
Edad (35-55) & 1 & & $<0,001$ \\
Edad (>55) & 1 & 5,1 & $<0,0001$ \\
& 3 & & $<0,0001$ \\
Bloque de variables socioeconómicas & & & $<0,05$ \\
Sí acceso & 1 & & $\mathrm{NS}$ \\
Profesionales & 1 & & $\mathrm{NS}$ \\
Independientes & 1 & & $<0,05$ \\
Obreros & 1 & 10,6 & $<0,0001$ \\
Inactivos & 1 & & $<0,0001$ \\
Dueñas de casa & 1 & 9,6 & $<0,0001$ \\
& 6 & 13,2 & $<0,0001$ \\
Bloque de variables psicosociales & & 17,6 & \\
Autoestima & 1 & 1 & \\
Autoeficacia & 2 & & \\
& & & \\
\hline
\end{tabular}

la relación simultánea de las variables psicosociales con el EVPS en que la autoestima y autoeficacia explican 17,6\% de su variación (el estado de salud percibido es eliminado del modelo de variables psicosociales puesto que no contribuye a explicar varianza adicional del EVPS).
Al estimar un modelo de regresión simultáneo con todos los determinantes próximos del EVPS (Tabla 5) resultó que ese modelo óptimo conformado por los predictores sexo, edad, ocupación, acceso a salud, autoestima y percepción de autoeficacia, explican 30\% la variación total del

Tabla 5. Resultados finales del ajuste de modelos de regresión a los datos sobre determinantes próximos del estilo de vida promotor de salud: M odelo óptimo

\begin{tabular}{|c|c|c|c|}
\hline Variables & $\begin{array}{l}\text { Parámetro } \\
\text { (B) }\end{array}$ & Standard error & $\mathrm{p}$ \\
\hline Sexo: (Hombres) & $-4,2$ & 2,1 & $<0,05$ \\
\hline Edad: (35-55 años) & 2,9 & 2,2 & NS \\
\hline (>55 años) & 8,3 & 2,4 & $<0,001$ \\
\hline Acceso a salud: (Sí) & 9,4 & 2,7 & $<0,001$ \\
\hline \multirow{5}{*}{$\begin{array}{ll}\text { Ocupación: } & \text { Profesionales } \\
& \text { Independientes } \\
& \text { Obreros } \\
& \text { Inactivos } \\
& \text { Dueñas de casa }\end{array}$} & 4,3 & 4,9 & NS \\
\hline & $-2,7$ & 3,1 & NS \\
\hline & $-3,3$ & 4,6 & NS \\
\hline & 4,4 & 3,1 & NS \\
\hline & 4,6 & 3,3 & NS \\
\hline Autoestima & 1,4 & 0,2 & $<0,0001$ \\
\hline \multirow[t]{2}{*}{ Autoeficacia } & 0,8 & 0,2 & $<0,0001$ \\
\hline & $\mathrm{R}^{2}=30,0$ & $\mathrm{~F}=13,5$ & $\mathrm{p}<0,0001$ \\
\hline
\end{tabular}


EVPS con un nivel de significación menor al uno por diez mil. El ajuste combinado de los bloques de variables sociodemográficas (sexo y edad), socioeconómicas (acceso a salud y ocupación) y de las orientaciones subjetivas (autoestima y autoeficacia) se constituye en el modelo óptimo final del EVPS. Estas seis variables explican 30\% de la variación total del estilo de vida promotor de salud del grupo de habitantes de Barrio Norte de Concepción.

En este modelo final, la autoestima y autoeficacia de las personas se revelan como las variables más importantes en explicar el estilo de vida promotor de salud. Niveles más altos de autoestima y autoeficacia están prácticamente asociados a puntajes más altos de EVPS, controlado por el sexo-edad, ocupación y estatus socioeconómico de las personas estudiadas. El control simultáneo en el modelo de características sociodemográficas (sexo y edad) y socioeconómicas (acceso a salud y ocupación) hace que el efecto cultural detectado para la autoestima y la autoeficacia sobre el estilo de vida en salud, sea significativo en lo teórico, robusto en lo estadístico y de relevancia estratégica en términos de generación de políticas sociales en salud.

\section{DisCUSIÓN}

Sólo un poco más de la mitad de los residentes del sector de Barrio Norte declara un estilo de vida promotor salud moderadamente adecuado. Esto indica que, a nivel local, aún se deberían intensificar las acciones dirigidas a la promoción, para producir un impacto mayor en la salud de las personas. Este resultado coincide con investigaciones realizadas en poblaciones latinoamericanas, americanas y afroamericanas, en las que se observa que los estilos de vida de las personas comprenden conductas negativas para la sa$\operatorname{lud}^{10,11}$. En estudios nacionales en población sana, se observó estilos de vida (EV) poco saludables en las personas estudiadas ${ }^{12-17}$. Estos hallazgos sugieren que las personas deben asumir un rol más activo en el desarrollo y mantención de conductas saludables, particularmente considerando el informe de la OMS, en que se demuestra que las enfermedades no transmisibles (asociadas a estilo de vida) fueron responsables de $60 \%$ de las muertes y de $43 \%$ de la carga de enfermedad global $^{18}$.

Se destaca en este estudio que los adultos maduros tienen más altos puntajes de EVPS que los adultos jóvenes y adolescentes, estos hallazgos encuentran apoyo en los estudios que sostienen que el EV parece mejorar con la edad, en respuesta tanto a cambios biológicos como psicosociales ${ }^{19-22}$. El hecho que se infiera que los adultos maduros son más responsables en salud que los adultos jóvenes, probablemente los adultos maduros están más conscientes de los riesgos que existen para su salud o a que ellos presentan episodios de enfermedad con más frecuencia, lo que los mantiene más en contacto con los sistemas asistenciales como apoyos sociales. Las variables sociodemográficas observadas en este estudio (sexo y edad), así como las socioeconómicas (acceso a salud y ocupación), no son susceptibles de modificar por intervención, pero son útiles para identificar y prestar mayor atención a personas que se encuentran en las categorías más vulnerables, por ejemplo: adultos jóvenes, obreros y sin acceso a salud.

Considerando que las variables autoestima y autoeficacia resultan muy importantes en predecir el EVPS, parece imprescindible considerarlas en forma simultánea en cualquier intervención que tenga esta finalidad. Estas variables han sido observadas también en otros estudios como factores importantes de la conducta ${ }^{23-25}$. Bandura indica que autoestima y percepción de autoeficacia (juicios de autovalía y de autocapacidad) no guardan relación uniforme; sin embargo, en la mayoría de las actividades que emprende el individuo cultiva su autoeficacia en aquellas que le dan sensación de autovalía ${ }^{26}$. Es importante mencionar que los adolescentes que se perciben saludables y con autovalía positiva son menos propensos a adoptar conductas fumadoras ${ }^{27,28}$. Del mismo modo, la percepción de sí mismos como saludables, la autovalía positiva y percibirse autoeficaces, son factores considerados motivadores en niñas adolescentes para participar en actividad física ${ }^{29-31}$.

Un aspecto de suma importancia a considerar en futuras intervenciones, es promover la autoeficacia y autoestima haciendo sentir a la persona valorada y exitosa en la realización de una determinada conducta o actividad. Esto requiere de retroa- 
limentación positiva y en general de estrategias de consejería para facilitar la adopción de conductas favorables en salud. También el aprendizaje desde las experiencias de otros puede ayudar a la persona a darse cuenta de que la conducta esperada es posible de lograr. Esto impone la incorporación de la persona a grupos de apoyo en donde pueda confrontar sus percepciones y logros con la de

\section{REFERENCIAS}

1. Organización Mundial de la Salud. Carta de Bangkok para la Promoción de la Salud en un Mundo Globalizado (2005). Disponible en www.who.org [Consultado en agosto de 2005]

2. Ministerio de Salud de Chile. Aporte a la Política Nacional de Prevención y Control de Enfermedades No Transmisibles en Chile (2002).

3. Ministerio de Salud de Chile. Promoción de la Salud (2003). Disponible en: www.minsal.cl [Consultado en junio de 2004]

4. Pender N, Murdaugh C, Parson M. Health Promotion in Nursing Practice. Jersey USA: Prentice Hall, 2002.

5. Pender N. Health Promotion In Nursing Practice. Stamford: Connecticut. Appleton and Lange, 1990.

6. Now C. Research Designs. Developing Research in Nursing and Health. New York USA: Ed. Springer Publishing Company, 1998; 19-29.

7. Walker S, Kerr M, Pender N, Sechrist K. A Spanish languaje version of the Health Promoting Lifestyles. Nurs Res 1990; 39: 268-73.

8. Rosenberg M. En Robinson J, Shaver P, Wrightman L. Measures of Personality and Social Psychological Attitudes. United States of America. Academic Press 1991; 121-3.

9. Schwarzer R, Babler. Spanish adaptation of the general perceived self-efficacy. Disponible en: www.RalfSchwarzer.de/ [Consultado el 30 de abril de 2002].

10. WANG H. Predictors of health promotion lifestyle among three ethnic groups uf elderly rural women in Taiwan. Public-Health Nursing 1999; 16: 321-8.

11. Monk T, Reynolds C, Kupfer D, Hoch C, Carrier J, otros $^{32-34}$. Debido a que este estudio sólo explica un tercio de la variación del EVPS, es importante continuar investigando otros factores que fueron omitidos en esta ocasión, tales como la percepción de beneficios, la percepción de barreras o las influencias situacionales en el EVPS, puesto que esas variables han resultado exitosas en estudios efectuados en otros contextos.

Houck P. Differences over the life span in daily life style regularity. Cronobiol Int 1997; 14: 295-306.

12. Marín P, Vilialobos A, Carrasco M, Kalache A. Resultados generales del Proyecto INTRA-OMS en Chile. Rev Méd Chile 2005; 133: 331-7.

13. Bello S, Soto M, Michaland S, Salinas J. Encuesta nacional de tabaquismo en funcionarios de salud. Rev Méd Chile 2004; 132: 223-32.

14. Miño C, Stiepovich J. Conductas Promotoras de Salud en Hipertensos. Revista Enfermería 2003; 124: 7-14.

15. Blümel M, Branco J, Castelo C, Roncaglolo M, BINFA E, SARRÁ S. Factores de riesgo cardiovascular en una cohorte de mujeres de edad media. Rev Méd Chile 2003; 131: 381-9.

16. Fagalde M, Solar J, Guerrero M, AтAlah S. Factores de riesgo de enfermedades crónicas no transmisibles en funcionarios de una empresa de servicios financieros de la Región Metropolitana. Rev Méd Chile 2005; 133: 919-28.

17. Ministerio de Salud - PUC. Encuesta Nacional de Salud. 2004. Disponible en: www.minsal.cl [Consultado en junio de 2004].

18. Pan American Journal of Public Health (2002). Informe de Salud. Disponible en: www.paho.org

19. Acton GJ, Malathum P. Basic need status and health-promoting selfcare Behavior in Adults. West J Nurs Res 2000; 22: 796-811.

20. Hulme P, Walker S, EfFle K, Jorgensen L, Mc Gowan M, Nelson J ET aL. Health promoting lifestyle behaviors of Spanish - speaking Hispanic adults. J Transcult Nurs 2003; 14: 244-54.

21. Walker S, Volkan K, Sechrist K, Pender N. Health Promoting Lifestyles of Older Adults: comparisons with young and middle-aged adults, correlates and patterns. ANS Adv Nurs Sci 1998; 11: 76-90. 
22. Brownson R, Eyler A, King A, Brown D, Shyu Y, SAшS J. Patterns and correlates of physical activity among US women 40 years and older. Am J Epidemiol 2002; 156: 945-53.

23. SCHWARZER R, RENNER B. Social- cognitive predictors of health behavior: action self-efficacy and coping self-efficacy. Health Psychol 2000; 19: 487-495. Disponible en: www.ncbi.nlm.gov [Consultado en noviembre de 2003].

24. MC Nichols SL. Social support and positive health practices. West J Nurs Res 2002; 24: 772-87. Disponible en: Medscape [Consultado el $17 \mathrm{de}$ noviembre de 2003].

25. Haquin C, Larraguibel M, Cabezas J. Factores protectores y de riesgo en salud mental en niños y adolescentes de la ciudad de Calama. Rev Chil Pediatr 2004; 75: 425-33.

26. Bandura A. Autoeficacia. En: Pensamiento y acción: Fundamentos sociales. España: Editorial Martínez Roca, 1987; 415-78.

27. MARTINEL A. An explanatory model of variables influencies health promotion behaviours in smoking and nonsmoking college students. Public Health Nursing 1999; 16: 263-9.

28. González L, Berger K. Consumo de tabaco en adolescentes: Factores de riesgo y factores protectores. Ciencia y Enfermería 2002; 8: 27-35.
29. Wu T, PENDER NJ. Determinants of physical activity among taiwanese adolescents: an application of the health promotion model. Res Nurs Health 2002; 25: 25-36.

30. Pender NJ, Bar-Or O, Wilk B, Mitchell S. Selfefficacy and perceived exertion of girls during exercise. Nurs Res 2002; 51: 86-91. Disponible en: http://wos.isitrial.com/CIW.cgi [Consultado el 19 de abril de 2002].

31. Cheng K, Cheng P, Mak K, Wong S, Wong Y, Yeung E. Relationships of perceived benefits and barriers to physical activity, physical activity participation and physical fitness in Hong Kong female adolescents. J Sports Med Phys Fitness 2003; 43: 523-9.

32. KerR M, LusK S, Ronis D. Explaining Mexican American worker's hearing protection use with the health promotion model. Nurs Res 2002; 51: 100-9. Disponible en: http://wos.isitrial.com/ CIW.cgi [Consultado el 19 de abril de 2002].

33. Berríos X, Bedregal P, Guzmán B. Costo-efectividad de la promoción de la salud en Chile: Experiencia del programa “iMírame!”. Rev Méd Chile 2004; 132: 361-70.

34. Cruz R, Noroño N, Fernández O, Cadalso R. Intervención educativa para la disminución de la agresividad en la conducta infantil. Rev Cubana Pediatr 2002; 74: 189-94.

Agradecimientos

Al Profesor Urcesino González Rubilar, un especial reconocimiento por su apoyo personal y asesoría estadística. 